\title{
Usage of Fuzzy Spatial Theory for Modelling of Terrain Passability
}

\author{
Alois Hofmann, ${ }^{1}$ Sarka Hoskova-Mayerova, ${ }^{2}$ and Vaclav Talhofer ${ }^{1}$ \\ ${ }^{1}$ Department of Military Geography and Meteorology, Faculty of Military Technology, University of Defence, Kounicova 65, \\ 66210 Brno, Czech Republic \\ ${ }^{2}$ Department of Mathematics and Physics, Faculty of Military Technology, University of Defence, Kounicova 65, \\ 66210 Brno, Czech Republic
}

Correspondence should be addressed to Sarka Hoskova-Mayerova; sarka.hoskova@seznam.cz

Received 14 September 2013; Accepted 10 October 2013

Academic Editor: Ferdinando Di Martino

Copyright (C) 2013 Alois Hofmann et al. This is an open access article distributed under the Creative Commons Attribution License, which permits unrestricted use, distribution, and reproduction in any medium, provided the original work is properly cited.

Geographic support of decision-making processes is based on various geographic products, usually in digital form, which come from various foundations and sources. Each product can be characterized by its quality or by its utility value for the given type of task or group of tasks, for which the product is used. They also usually have different characteristics and thus can very significantly influence the resulting analytical material. The aim of the paper is to contribute to the solution of the question of how it is possible to work with diverse spatial geographic information so that the user has an idea about the resulting product. The concept of fuzzy sets is used for representation of classes, whose boundaries are not clearly (not sharply) set, namely, the fuzzy approach in overlaying operations realized in ESRI ArcGIS environment. The paper is based on a research project which is being solved at the Faculty of Military Technologies of the University of Defence. The research deals with the influence of geographic and climatic factors on the activity of armed forces and the Integrated Rescue System.

\section{Introduction}

Geographic support for decision-making processes is based on various geographic products, usually in digital form, which come from various foundations and sources. Each product can be characterized by its quality $[1,2]$ or by its utility value for the given type of task or group of tasks, for which the product is used $[3,4]$. In both cases, among others also positional and thematic accuracy are evaluated either as an exactly given value, for example, mean square position error, probable error, and so forth, or as a level of fulfilment of user's requirements expressed in percentage [5]. Decision-making processes are based on multicriteria decision-making, in which many factors are involved [6]. By a suitable combination of various factors, analytical products are created. They are the base for answering questions such as "What happens if...?" That is the reason why various foundations are used for geographic support. They also usually have different characteristics and thus can very significantly influence the resulting analytical material. The aim of the paper is to contribute to the solution of the question of how it is possible to work with diverse spatial geographic information so that the user has an idea about the resulting product. The paper is based on a research project which is being solved at the Faculty of Military Technologies of the University of Defence. The research deals with the influence of geographic and climatic factors on the activity of armed forces and the Integrated Rescue System.

Within the work on solution of the project, the problem solving team continued with development of models of influences of geographical and climatic factors on terrain passability. In the evaluated period, the team focused on development of the theory of models, on ways of visualization of vagueness of results caused by inhomogeneity of the support data, combining their characteristics within the performed analyses, and so forth. For these purposes, fuzzy logic was used. 


\section{Vagueness of Spatial Information}

Standardly used types and procedures of geoprocedural analyses suppose that the used data are created with explicit, sharp boundaries that restrict the position of the individual objects.

The technical quality of position setting of such boundaries is usually given by the above-mentioned criteria, such as mean square positional error of its determination. The technical quality is based on measurement methods or digitalization of foundation.

Boundaries of many geographical elements are, however, only a result of human perception and not of a real matter of things. Even in case of existence of a real discrete boundary, the boundary line may be inaccurate due to vagueness of data or their interpretation. Vegetation or soil types are typical examples of geographical elements where there are no clear natural boundaries in space. Traditional classifications fail completely here. However, spatial units are usually represented by sharp boundaries. Brown and Heuvelink [7] suggest 2 types of uncertainty that have a special importance in GISthematic and spatial vagueness. In the first case we are not able to confirm occurrence of the given topic in the given place, and in the second case it is an inability to find an exact placement of the given topics. The formal conceptual model of uncertainty is useful to create for effective GIS utilization in the decision-making process [8]. Several authors tried to classify uncertainty [9-12], but the most used classification can be found in the standard for spatial data transfer [13].

As people both reason and make decisions with uncertain geospatial data every day, it is important to understand the complexity of uncertainty, how it propagates through each dataset, and how to best visualize uncertainty to support reasoning and decision-making [14-18].

Spatial and thematic data should not be evaluated independently. The way to solve these problems is to use the theory of "soft classifications", among which also "fuzzy" approaches belong. Fuzzy logic is quite frequently used for uncertainty of GIS data expression in the last years and is based on fuzzy theory [19-21].

Fuzzy sets together with the theory of fuzzy logic offer a scope for processing of predicates, whose level of probability is given in degrees ("true to certain degrees") and uncertainty is also expressed gradually. The concept of fuzzy sets deals with representation of classes, whose boundaries are not clearly (not sharply) set. When sharp boundaries separating the set from the surroundings are missing, a problem of unambiguous setting of an element belonging to a set and to its complement appears [22, 23].

Fuzzy files are then files or classes that do not have sharp limitation. With spatial data it means that at considered places the transition between the membership and nonmembership in a file is gradual. The fuzzy file then can be characterized by fuzzy levels of membership in an interval from 0.0 to 1.0 which expresses a gradual growth of membership from nonmembership up to the full membership. It can be defined with the help of the membership function.

In the environment of GIS three basic types of geoelements are usually defined: points, lines and areas (polygons).
When we use linesm and areas we sometimes ask a question of how to delimit boundaries of the given geoelement. If there is an area layer which captures ecological stability of the given area, then there are only two possibilities of how to express stability: stable or unstable. This classification is very difficult and it depends on the person that decides and on the concrete area.

One of the basic characteristics that can be defined when creating and saving geographical objects is topology. Topological relations characterize the relative placement of two spatial objects with respect to their mutual positionfor example, if they touch, overlay, or contain one another. In GIS they are important especially for a definition of spatial questions and selections and they play an important role when language SQL is used. In case of fuzzy spatial objects, however, traditional topologic predicates fail and their fuzzy variants come into consideration; they are able to answer inquiries such as the following.

(i) Do areas A and B overlay at least a bit?

(ii) Does area A partly contain area B?

(iii) Which areas are partly inside area $\mathrm{B}$ ?

The fact that belonging of an element to a fuzzy topological predicate is expressed by a set $[0,1]$, however, complicates its direct usage in language SQL and thus possible spatial inquiries.

\section{Fuzzy Overlay}

A concrete possibility of usage of the fuzzy approach is the application in overlaying operations that can be realized in the environment of ArcGIS [24]. For this type of operations it is nowadays possible to use tools of map algebra as well as already implemented tools in the extension of Spatial Analyst. Fuzzy logic in the so-called "Overlay Analysis" is based on two fundamental steps.

The first one is the so-called "fuzzification" or "fuzzy membership," in other words the process of implementing values into fuzzy sets, and the second step is the actual analysis-overlay of these sets. It is possible to use several types of fuzzification functions, from a linear to Gaussian function, according to the type of value distribution.

Relations among created fuzzy sets are then analysed by means of fuzzy overlaying operations, such as FuzzyAnd and FuzzyOr. The detailed description can be found for example, in the help file of ArcGIS Help.

Practical approaches of realization of usage of the "fuzzy" approach in analysis of geospatial data were the subject of development of procedures of finding an optimal route with the help of map algebra, which was suggested as one of the results of the mentioned project. It was testing of data that showed positional vagueness.

\section{Terrain Passability}

A very frequent task in the decision-making process in armed forces is to evaluate the possibility of vehicle movement in a terrain. This task is usually called Cross-Country Mobility 
(CCM) in military language. The main goal of CCM is to evaluate the impact of geographic conditions on movement of vehicles in terrain $[25,26]$. For the purpose of classification and qualification of geographic factors of CCM, it is necessary to determine

(i) particular degrees of CCM,

(ii) typology of terrain properties evaluated by the kind of vehicles used,

(iii) geographic factors and features with significant impact on CCM.

As a result of the geographic factors impact evaluation we get three CCM degrees: passable terrain, passable terrain with restrictions, or impassable terrain.

The impact of a geographic factor can be evaluated as a coefficient of deceleration " $C_{i}$ " on the scale of 0 to 1 . The coefficient of deceleration shows the real (simulated) speed of vehicle $v$ in the landscape in the confrontation with the maximum speed of a given vehicle $v_{\max }$. The impact of all $n$ geographic factors can be expressed by the following formula:

$$
v=v_{\max } \prod_{i=1}^{n} C_{i}, \quad n=1, \ldots, \mathbb{N} .
$$

The main coefficients of deceleration are listed in Table 1.

In case of searching for an optimal route within the project, a procedure of fuzzification for calculation of coefficient of deceleration $C_{3}$ (influence of soil and ground cover) was looked for. The initial data layer was a raster layer of coefficient $C_{3}$, where there are 3 values for passable (1), passable with difficulty (0.5), and impassable terrain (0). From the point of view of the input data-that is, area of soil types and kinds-a presumption was introduced that the boundary of passability will not be sharp according to the original data basis but it will change its value in the distance of $100 \mathrm{~m}$ to both sides. Based on this presumption, fuzzy sets were created with the use of several raster techniques and their overall influence was analysed.

In the next step, possibilities of analysis of several resulted sets were tested by their mutual interaction (overalls) through various methods. The solution of the problem, however, was not finished in such a way so that it was possible to prove the change of course of the found route due to vagueness. The authors suppose that the stated results will be further developed in the years to come when solving the project and their successive transfer to models with vagueness consideration. The way of solution is discussed in the following paragraphs.

\section{Development of Creation of CCM Models Using Fuzzy Logic}

Six basic models were created for searching of the way. With the help of these models it is possible to create a so-called Cost Map, which is a raster file that is the basic input information for creation of the file of the searched route. The cost map is made by application of overlaying operations or in this case with the use of the so-called map algebra that provides tools
TABLE 1: Main coefficients of deceleration.

\begin{tabular}{ll}
\hline Basic coefficient & Geographic signification and impact \\
\hline$C_{1}$ & $\begin{array}{l}\text { Terrain relief (gradient of terrain relief and } \\
\text { microrelief shapes) }\end{array}$ \\
$C_{2}$ & Vegetation cover \\
$C_{3}$ & Soils and soil cover \\
$C_{4}$ & Weather and climate \\
$C_{5}$ & Hydrology \\
$C_{6}$ & Built-up area \\
$C_{7}$ & Road network \\
\hline
\end{tabular}

for working with raster files. To set the overall coefficient of deceleration when moving through terrain, relations stated in the elaborated methodology were used.

Models for calculation of individual coefficients were compiled with the help of basic operations with raster data by means of implemented tools of the so-called Spatial Analyst, which is an extension of ArcGIS system, as well as using a tool of the so-called map algebra, that is, a set of operators and functions for work with raster data.

Raster layers were used as input data. The layers were created by calculation (e.g., a high raster model) or by a conversion of vector data according to the appropriate attributes. This data base was created as explicit data with clearly defined objects and their boundaries.

Another step when solving CCM problem is the introduction of some uncertainty causing a greater activity in decision-making about the use of the gained results. For each coefficient of deceleration, a new process model was made, in which principles of fuzzy logic were applied. For solution of the individual models, various approaches had to be used with respect to the character of input data and result that shall be reached.

5.1. Creation of Models for the Individual Coefficients. In the following text are briefly discussed procedures of calculation of the individual coefficients of deceleration that were realized in the environment of ArcGIS.

5.1.1. Coefficient of Deceleration due to Elevation of Terrain. Layers of relief and objects of microrelief are taken as source data. The compiled model is created from a layer of relief that was calculated with the help of raster interpolation and layers of point, line, and polygons.

In case of raster relief, the value of height is determined for each individual pixel of high area. In this case for its fuzzification we used data about the height range of the height model and setting of boundary for determination of impassability for the given means of transport.

For other layers the procedure is based on several substeps. The first step is the conversion of the layer in a raster and its editing. Data about height and depth of an object were used to convert height objects to a raster format. During the conversion of a vector into a raster it happens that the individual objects are converted into a set of pixels with a value of an attribute, according to which the conversion 
is realized (e.g., hgt, which is a height of an object). All surrounding pixels are marked with attribute NoData. These pixels would not enter into the calculation of the cost map not only of this but also of all other overlaying raster layers. The editing is testing of individual pixels for attribute NoData (by function IsNull) and assigning value 0 to all found pixels. Thus it is secured that all pixels outside height objects have a zero value which is later used when creating the "Cost Map". This procedure is applied also for other input layers.

The second step is a conversion of values "integer" raster to values in a real domain so that the operation of "fuzzification" could be initiated, that is, implementing of values of the raster into a "fuzzy set" with the help of one of the "fuzzification" functions. The input raster was in these cases divided by the value 10.0 so that a raster with real values was created. For verification of the "fuzzy" approach to this problem, only linear function was used so far in all phases of calculation.

The third step of the solution is the use of "fuzzy" sets for application of vagueness in the position of the given types of objects. This problem is solved with the help of calculation of "Euclidean" distance for each type of objects. Placing values of distance into "fuzzy" sets uses once again a linear function with setting of various values of maximum distance. It depends on the values of four classes of accuracy that the database creator gives [27]:

(i) $<0.5$ meter for geodetic points,

(ii) $<3$ metres for stable objects,

(iii) $<10$ metres for other objects,

(iv) $<20$ metres for unstable objects.

Apart from these values it is necessary to consider also the size of a pixel that is used for conversion of vector drawing to a raster. The above stated values make sense in case the size of a pixel is maximally 1 meter.

For comparison, cost maps were created with a pixel value of 5 metres and fuzzification of distance was in one case chosen the same as the size of a pixel (which actually represents a sharp boundary and thus it does not get blurred) and in another case the distances are chosen mostly as $100 \mathrm{~m}$ besides calculation of factors $C_{6}$ and $C_{7}$ where the value was reduced to $30 \mathrm{~m}$ with respect to the character of objects.

The last step for most calculated factors is a combination of the gained results with the help of the so-called "Fuzzy Overlay," that is, weighted "fuzzy" overlay of the individual raster layers (within calculation of factor $C_{1}$ ).

Overlay can be realized with the use of various logical operations, in the case of logical sum "OR" that made sure the highest value from the overlaying rasters was written into the resulting pixel; in most cases it was value " 1 " representing basically impassable terrain (see Figures 1 and 2 in detail).

5.1.2. Coefficient of Deceleration due to Vegetation. In case of vegetation, the type of vegetation is significant information. If it is vegetation with grown trees, information about trunk cross-section and spacing between trees are taken as parameters. For fuzzification, such a procedure was chosen that

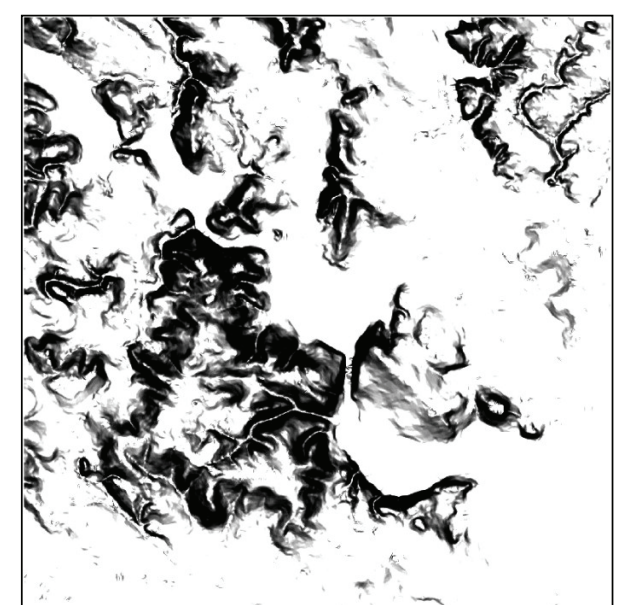

FIgURE 1: Visualization of calculation results of coefficient $C_{1}$.

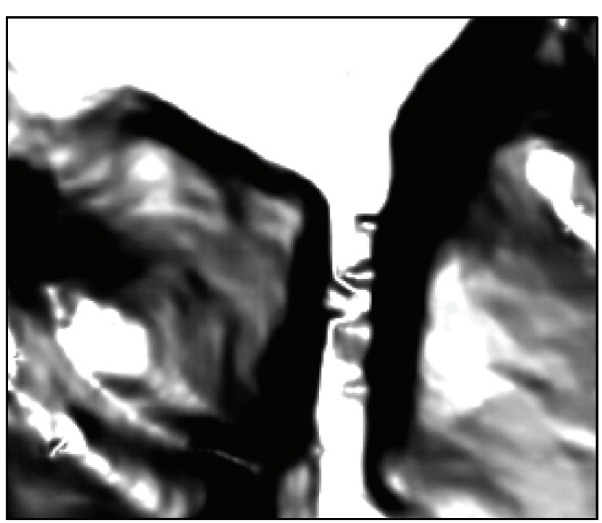

Figure 2: Visualization of calculation results of coefficient $C_{1}$-detail.

stems from classification of vegetation according to a value of these parameters and introduction of geometric vagueness of boundaries of in this way classified vegetation. The result is a layer, in which appropriate values are assigned to areas and their boundaries are blurred with the help of a distance function and their values change depending on the type of fuzzification method (Figures 3 and 4).

5.1.3. Coefficient of Deceleration due to Soils. For calculating this coefficient, attributes soil type, soil kind, and matrix were used. Using logical operators helped to determine passability of soils in four values $(1,2,3$, and 4). For fuzzification a procedure that is based on these values was used together with application of the general steps stated before, with the difference in vegetation lying in the method of creation of vague boundaries of the individual classes of soil polygons. In this case, these classes had to be transformed into independent layers for which distance rasters for blurring of their boundaries were calculated. Unlike the previous values of accuracy that were applied for all other factors of deceleration, boundaries for blurring with soils were chosen to be $100 \mathrm{~m}$ with respect to the character of this geographical element. The calculated raster entered into other steps of 


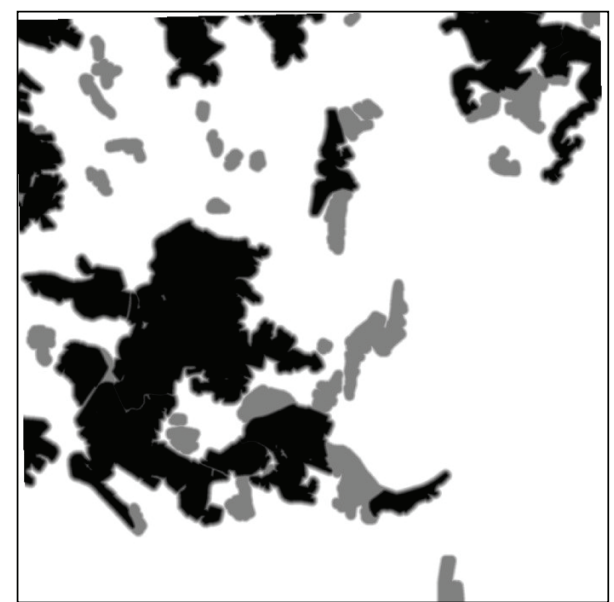

FIgURE 3: Visualization of calculation results of coefficient $C_{2}$.

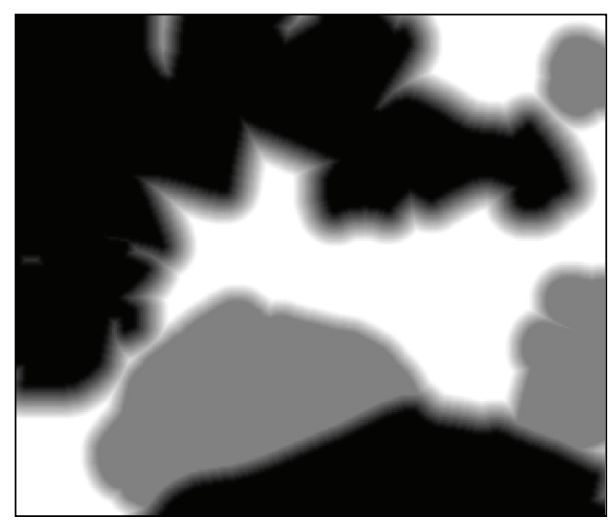

FIgURE 4: Visualization of calculation results of coefficient $C_{2}$-detail.

calculation depending on the set value, especially by keeping of the already calculated fuzzified value for various levels of passability. This is realized with the help of multiplication process of the individual fuzzy distance raster by the fuzzy raster of passability.

The result is a raster with different values of passability and blurred boundaries of polygons (see Figures 5 and 6).

5.1.4. Coefficient of Deceleration due to Water Courses and Areas of Water. The calculation of this coefficient is rather complicated with respect to the fact that there should be quite a lot of input layers of various characters entering the calculation. When modelling, a reduced scale of input layers was used with regards to the fact that appropriate attributes were not available (e.g., character of bottom or banks). For creation of the model, a wider range of processes was used and during the calculation also more semiresults occurred that entered into the calculation of the final overlaying fuzzy operation.

The evaluation itself of water objects was different with respect to its modelling (lines object for streams and narrow rivers polygons for wide rivers, lakes, and ponds, etc.). For line water, attributes such as depth and width of the course

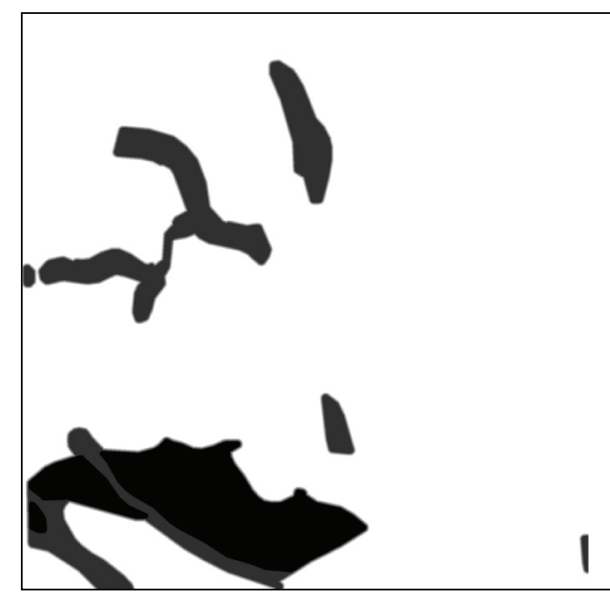

FIgURE 5: Visualization of calculation results of coefficient $C_{3}$.

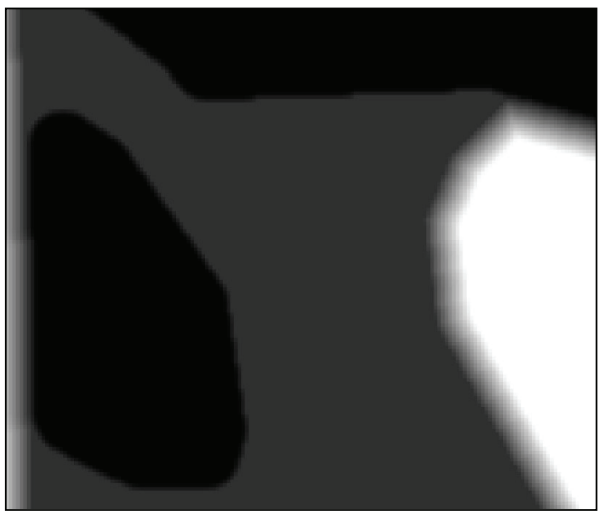

FIGURE 6: Visualization of calculation results of coefficient $C_{3}$-detail.

were taken from the value of pixels creating a raster line, for areal; however, the width is given by the total area of pixels creating the area of water. Depth is then written into each pixel of the object in the same value.

For distance calculations, distance rasters for flowing and slack water were calculated separately. The result of the calculation can be visualized similarly.

5.1.5. Coefficient of Deceleration due to Built-Up Area. The solution of calculation of the model of a built-up area is divided into two parts. The first part is based on a presumption that all buildings are impassable and only the principle of vagueness of boundaries or position of individual objects in a layer of building is applied. The second part works with the possibility of different passability through a built-up area. It is based on attributes from a layer of block built-up area and it counts with different passability for various types of a built-up area.

5.1.6. Coefficient of Deceleration on Communications. The problem of calculation of coefficient of deceleration for communications is made difficult by the fact that for most motorized means of transport communications are well 


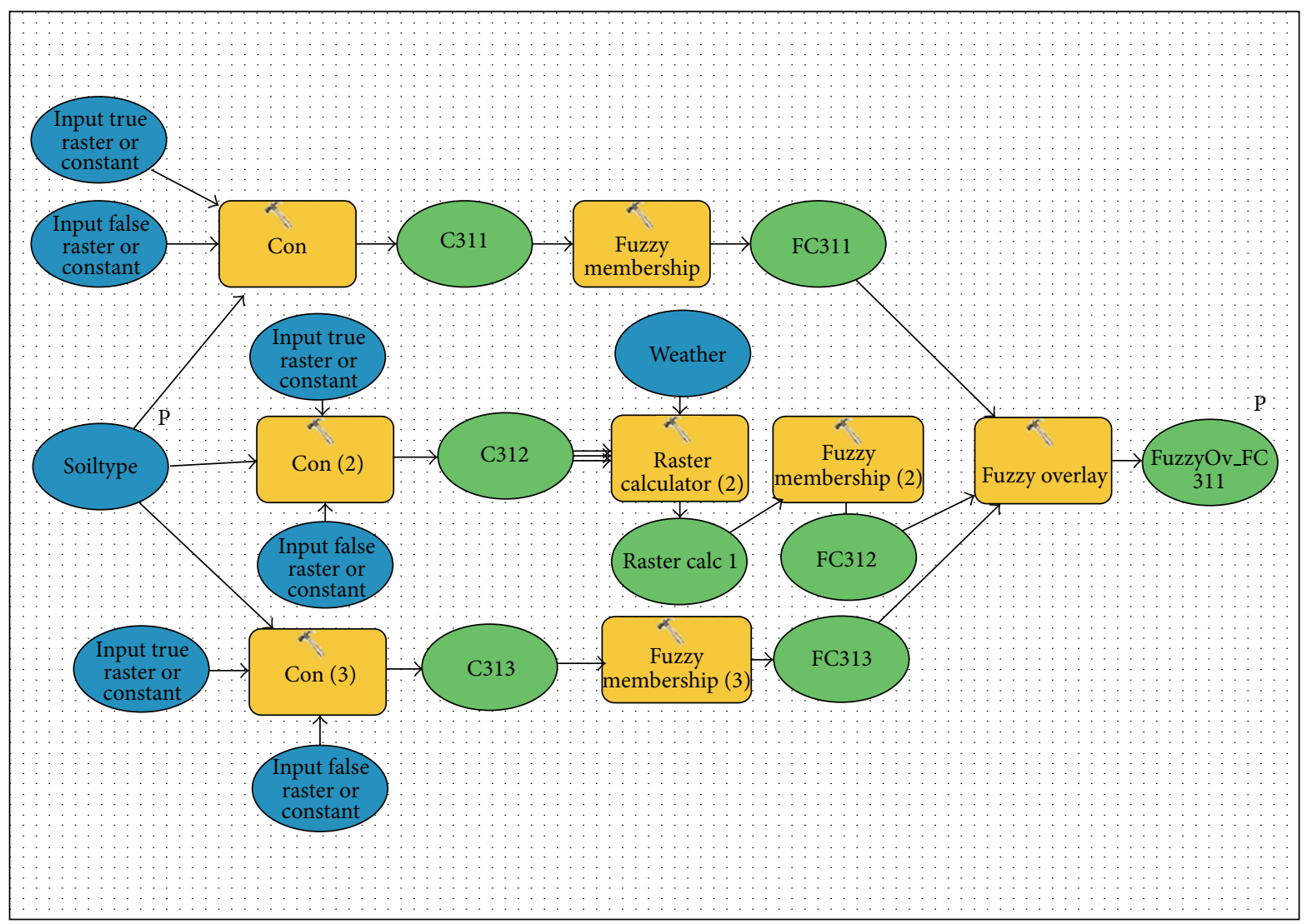

FIGURE 7: Demonstration of a model for calculation of deceleration coefficient created in the environment of ModelBuilder ArcGIS.

passable and this must be considered for adaptation of the calculation in such a way that the value of communication passability was more convenient for the object of communication (only communications over land are chosen) rather than for the surrounding terrain. This makes sure that for transport the route over communication is chosen and if the destination lies in a free terrain, then the route is calculated with consideration of all coefficients already on terrain. A tool of reclassification was used for calculation. In this case it is more convenient and ensures a suitable distribution of values of the attribute of transportation usage of the communication. The uncertainty in position is once again ensured by a distance raster.

The presented results are only the beginning of the solution of the complete problem. Demonstration of the fuzzy model is shown in Figure 7. Further solution will go on in two directions. They are the use of other fuzzification functions on one hand and optimization of the choice of distance, that is, vagueness level for the individual types of objects, on the other. The third problem is a determination of the final, common coefficient of deceleration and creation of a cost map. Once the third problem is successfully solved, testing of the suggested combination of parameters of fuzzification and a suggestion of methodology of decision-making with the use of fuzzy cost map will take place.
5.2. Verification of Results. The resulting cost map is only a kind of a foundation for making a decision. With the help of this map it is possible to search for an optimized route for the given vehicle type from place $A$ to place $B$ and judge to which extent the calculated route is suitable for the considered vehicle.

Within the solution of the project, two variants of calculation of the route for a military heavy vehicle Tatra 815 were considered [28]. One of them used a cost map derived without using fuzzy principles, and the other used these principles. With regard to the possibility to verify the calculation results, the urbanized area of the city of Brno and its closest surroundings were chosen.

With the help of these maps, routes of the stated vehicle between identical points were calculated. The results of the calculated routes are shown in the figures. With respect to the fact that for calculations reinforced communications were set as priorities, there are no significant differences in the calculated routes. Both of the calculated routes were then verified directly in real terrain. The aim of this verification was to find out to which extent the calculation itself is influenced by the use of the fuzzy method and how the use-or nonuse-of the fuzzy method will affect the given calculations. Another aim of the verification was to verify the quality of the used input data. Based on the results 
of the terrain research it is possible to say that in city agglomerations where there is a sufficient net of quality reinforced communications using fuzzy principles is almost unnecessary and if sufficient quality input data are used, it is more effective to use the method of sharp boundaries for the calculation of a cost map.

Another situation, however, can happen outside urbanized areas where the net of reinforced communications is scarce and where there are a lot of forests, fields, and so forth. This fact occurred only on the edge of the researched area. That is the reason why the team for solution will focus on this type of countryside in the following steps.

\section{Acknowledgment}

The work presented in this paper was supported within the project for Development of Military Geography and Meteorology and for Support for Mathematical and Physical Research supported by the Ministry of Defence of the Czech Republic.

\section{References}

[1] ISO, ISO 19113-Geographic Information-Quality Principles, International Organization for Standardization, 2002, http:// www.iso.org/iso/home/storecatalogue_tc/catalogue_detail .htm?csnumber $=26018$.

[2] ISO, ISO 19138-Geographic Information-Data Quality Measures, Interational Organization for Standardization, 2006, http://www.iso.org/iso/catalogue_detail.htm? csnumber=32556.

[3] V. Talhofer and A. Hofmann, "Possibilities of evaluation of digital geographic data quality and reliability," in Proceedigs of the 24th International Cartographic Conference, the World's GeoSpatial Solutions, pp. 1-11, ICA/ACI, Santiago de Chilie, Chile, 2009.

[4] V. Talhofer, S. Hoskova-Mayerova, and A. Hofmann, "Improvement of digital geographic data quality," International Journal of Production Research, vol. 50, no. 17, pp. 4846-4859, 2012.

[5] V. Talhofer, S. Hoskova, V. Kratochvil, and A. Hofmann, "Geospatial data quality," in International Conference on Military Technologies (ICMT '09), pp. 570-578, Univerzita obrany, Brno, Czech Republic, 2009.

[6] V. Kovařík, "Use of spatial modelling to select the helicopter landing sites," Advances in Military Technology, vol. 8, no. 2, pp. $1-10,2013$.

[7] J. D. Brown and G. B. M. Heuvelink, "The data uncertainty engine (DUE): a software tool for assessing and simulating uncertain environmental variables," Computers \& Geosciences, vol. 33, no. 2, pp. 172-190, 2007.

[8] R. Abbaspour, R. Mahmoud, K. Delavar, and B. Reihaneh, “The issue of uncertainty propagation in spatial decision making," in Proceedings of the 9th Scandinavian Research Conference on Geographical Information Science (ScanGIS '03), K. V. Tveite, Ed., pp. 57-65, Department of Surveying, Helsinki University of Technology, Espoo, Finland, 2003.

[9] M. F. Goodchild, S. Guoqing, and Y. Shiren, "Development and test of an error model for categorical data," International Journal of Geographical Information Systems, vol. 6, no. 2, pp. 87-104, 1992.
[10] G. Hunter, Handling Uncertainty in Spatial Database [Ph.D. thesis], Department of Surveying and Land Information, University of Melbourne, Melbourne, Australia, 1993.

[11] O. Křemenová, Fuzzy Modeling of Soil Maps, University of Technology, Helsinky, Finland, 2004.

[12] P. Kubíček and Č. Šašinka, "Thematic uncertainty visualization usability-comparison of basic methods," Annals of GIS, vol. 17, no. 4, pp. 253-263, 2011.

[13] NIST, Spatial Data Transfer Standard (FIPS 173), National Institute of Standards and Technology, US Department of Commerce, Washington, DC, USA, 1992.

[14] P. D’Amico, F. Di Martino, and S. Sessa, "A GIS as a decision support system for planning sustainabel mobility in a casestudy," in Multicriteria and Multiagend Decision Making with Applications to Economics and Social Sciences, A. Ventre, A. Maturo, S. Hoskova-Mayerova, and J. Kacprzyk, Eds., Studies in Fuzziness and Soft Computing, pp. 115-128, Springer, Berlin, Germany, 2013.

[15] F. De Felice and A. Petrillo, "Decision making analysis to improve public participation in stategic energy production management," in Multicriteria and Multiagend Decision Making with Applications to Economics and Social Sciences, A. Ventre, A. Maturo, S. Hoskova-Mayerova, and J. Kacprzyk, Eds., Studies in Fuzziness and Soft Computing, pp. 129-142, Springer, Berlin, Germany, 2013.

[16] N. Gershon, "Visualization of an imperfect world," IEEE Computer Graphics and Applications, vol. 18, no. 4, pp. 43-45, 1998.

[17] A. MacEachren, "Visualizing uncertain inforamtion," Cartographic Perspectives, vol. 13, pp. 10-19, 1992.

[18] J. Smith, D. Retchless, C. Kinkeldey, and A. Klippel, "Beyond the surface: current issues and future irections in uncertainty visualization research," in Proceedigs of the 26th International Cartographic Conference, pp. 1-10, ICA, Dresden, Germany, 2013.

[19] B. Ahmad and A. Kharal, "Fuzzy sets fuzzy s-open and s-closed mappings," Advances in Fuzzy Systems, vol. 2009, Article ID 303042, 5 pages, 2009.

[20] F. Di Martino, V. Loia, and S. Sessa, "Fuzzy transforms method in prediction data analysis," Fuzzy Sets and Systems, vol. 180, no. 1, pp. 146-163, 2011.

[21] L. A. Zadeh, "Fuzzy sets," Information and Control, vol. 8, no. 3, pp. 338-353, 1965.

[22] F. Di Martino and S. Sessa, "Spatial analysis and fuzzy relation equations," Advances in Fuzzy Systems, vol. 2011, Article ID 429498, 14 pages, 2011.

[23] I. Cristea and S. Hoskova, "Fuzzy pseudotopological hypergroupoids," Iranian Journal of Fuzzy Systems, vol. 6, no. 4, pp. 11-19, 2009.

[24] ESRI, “User documentation," Copyright ( $1995-2013$ Esri.

[25] M. Rybansky, Cross-Country Movement, the Imapct and Evaluation of Geographic Factors, Akademické nakladatelství CERM, s.r.o. Brno, Brno, Czech Republic, 1st edition, 2009.

[26] M. Rybansky and M. Vala, "Relief impact on transport," in International Conference on Military Technologies (ICMT '09), pp. 551-559, University of Defence, Brno, Czech Republic, 2010.

[27] MoD-GeoS, Catalogue of the Topographic Objects DMU25, Ministry of Defence of the Czech Republic, Geographic Service, Dobruska, Czech Republic, 7.3 edition, 2010.

[28] Tatra, Tatra is the solution. (Tatra, a.s.), TATRA, 2010, http:// partners.tatra.cz/exter_pr/vpnew/typovy_listprospekt.asp? kod=341\&jazyk=CZ. 

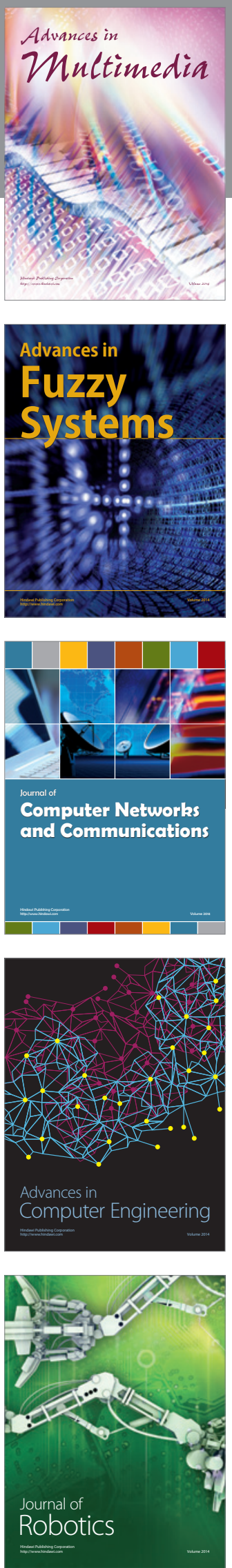

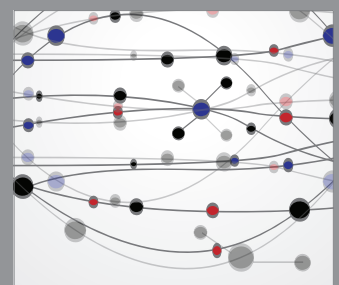

The Scientific World Journal
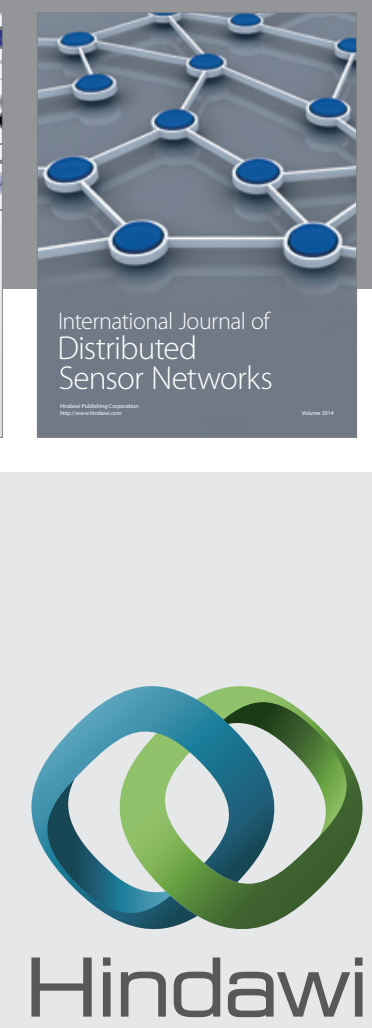

Submit your manuscripts at

http://www.hindawi.com
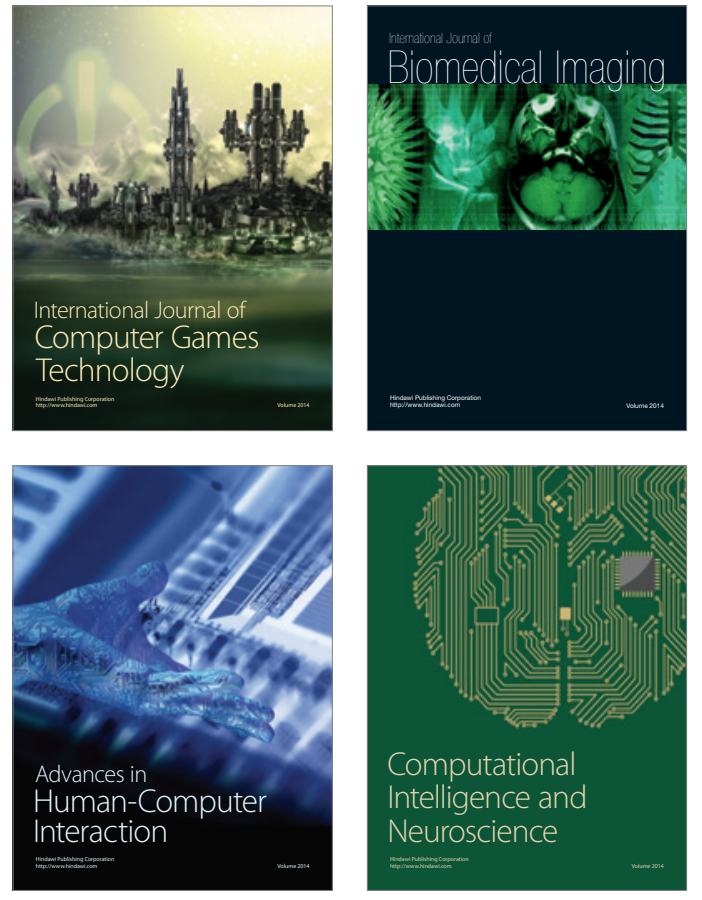
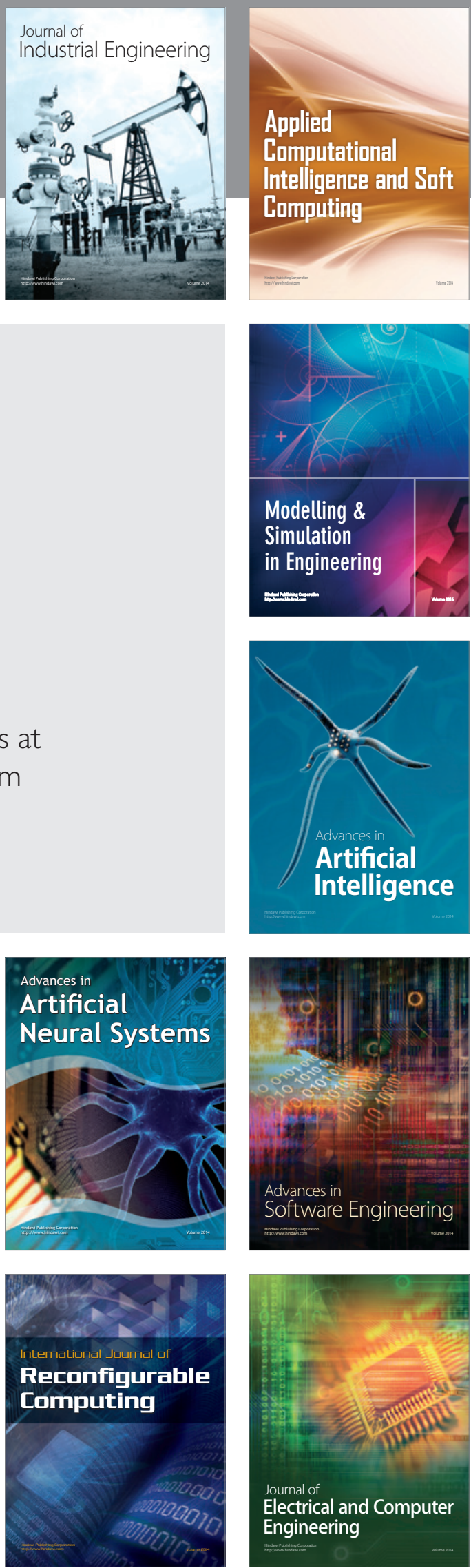